Quim. Nova, Vol. 35, No. 9, 1814-1818, 2012

\title{
CARACTERIZAÇÃO DE CHÁS DE GENÓTIPOS DE Lippia gracilis Schauer ATRAVÉS DE PERFIL CROMATOGRÁFICO POR CLAE-DAD COMBINADO COM ANÁLISES QUIMIOMÉTRICAS
}

\author{
Vilma Menezes de Jesus Prado, Valéria Regina de Souza Moraes* e Paulo Cesar de Lima Nogueira \\ Departamento de Química, Universidade Federal de Sergipe, 49100-000 São Cristovão - SE, Brasil \\ Elizangela Mércia Oliveira Cruz e Arie Fitzgerald Blank \\ Departamento de Agronomia, Universidade Federal de Sergipe, 49100-000 São Cristovão - SE, Brasil \\ Edenir Rodrigues Pereira-Filho \\ Departamento de Química, Universidade Federal de São Carlos, CP 676, 13560-970 São Carlos - SP, Brasil \\ Lúcia Regina Rocha Martins \\ Departamento de Química e Biologia, Universidade Tecnológica Federal do Paraná, 81280-340 Curitiba - PR, Brasil
}

Recebido em 26/3/12; aceito em 4/6/12; publicado na web em 24/8/12

\begin{abstract}
CHARACTERIZATION OF TEAS FROM Lippia gracilis Schauer GENOTYPES BY HPLC-DAD CHROMATOGRAPHIC PROFILE COMBINED WITH CHEMOMETRIC ANALYSES. In order to evaluate the effects of environmental factors on the content of secondary metabolites, the chemical profiles of infusions from leaves of seven genotypes of Lippia gracilis Schauer, sourced from two locations (Sergipe and Bahia state) and collected during different seasons: summer (with and without irrigation) and winter, were determined by HPLC-DAD. The fingerprint chromatograms were analyzed by PCA to evaluate similarities and differences among the samples. Results revealed differences among genotypes collected and cultivated under the same conditions, suggesting that three genotypes have greater resistance to drought conditions.
\end{abstract}

Keywords: Lippia gracilis Schauer; HPLC-DAD; chemometric analyses.

\section{INTRODUÇÃO}

A família Verbenaceae J. St.-Hil. ocorre em praticamente todos os ecossistemas terrestres, compreendendo cerca de 100 gêneros e 2000 espécies, distribuídas em regiões tropicais e subtropicais, principalmente nas zonas temperadas do hemisfério sul. ${ }^{1}$

O gênero Lippia inclui cerca de 200 espécies de ervas, arbustos e pequenas árvores distribuídas por todos os países da América do Sul e Central e pela África tropical, com cerca de 120 espécies ocorrendo no Brasil. Algumas espécies de Lippia são caracterizadas pela presença de óleos essenciais, ${ }^{2}$ geralmente possuindo uma fragrância forte e agradável, apresentando altos efeitos antimicrobianos sobre vários micro-organismos, devido à presença dos monoterpenos fenólicos, timol e carvacrol. ${ }^{3}$

Lippia gracilis Schauer é uma planta nativa do nordeste brasileiro encontrada, principalmente, nos estados da Bahia, Sergipe e Piauí. No sertão nordestino encontra-se a altitudes de cerca de $450 \mathrm{~m}$, sendo, popularmente conhecida como cidreira da serra; enquanto que no Agreste encontra-se a altitudes em torno de 960 m, sendo conhecida popularmente como alecrim-da-chapada ou alecrim-de-tabuleiro. ${ }^{4} \mathrm{As}$ comunidades tradicionais da Caatinga, região semiárida do nordeste brasileiro, utilizam suas folhas para o tratamento de infecções de garganta, doenças cutâneas, úlceras, afecções na vagina, acne, caspa, queimaduras, feridas, sinusite, bronquite, congestão nasal, dor de cabeça, icterícia e paralisia. ${ }^{5,6}$

Ao nosso conhecimento, há apenas um único relato sobre os constituintes fixos desta espécie, ${ }^{7}$ sendo que os demais trabalhos se concentram nos estudos sobre a composição química do óleo essencial e sua atividade biológica. ${ }^{2,8}$ No entanto, há diversos estudos fitoquímicos sobre outras espécies deste gênero, os quais descrevem o isolamento de várias substâncias, destacando-se iridoides glicosilados, feniletanoides glicosilados, saponinas triterpênicas, naftoquinonas,

*e-mail: valrsmoraes@uol.com.br flavonoides, flavonoides glicosilados, lignana, sesquiterpenos e triterpenos. ${ }^{2,9}$

De forma geral, o perfil cromatográfico de um extrato vegetal pode ser considerado representativo da complexidade química dessa amostra, guardadas as devidas ressalvas em relação ao método específico escolhido, bem como as limitações do detector utilizado. Diversos trabalhos na literatura mencionam o uso deste perfil cromatográfico como um método analítico que pode avaliar a relação entre a informação química e as características de cada amostra vegetal, tais como a diferenciação entre espécies botanicamente semelhantes, a variabilidade entre plantas coletadas em diferentes locais geográficos, sob diferentes condições climáticas e de cultivo. ${ }^{10-13}$

Para identificar semelhanças e diferenças entre as amostras analisadas usando a informação química dada pelo perfil cromatográfico foi realizada a análise de componentes principais (ACP), que consiste numa transformação da matriz de dados com o objetivo de representar as variações presentes em muitas variáveis, através de um número menor de "fatores" sem desconsiderar informações relevantes. ${ }^{14,15}$

Assim, este trabalho teve como objetivo a discriminação de chás de sete genótipos de Lippia gracilis Schauer oriundos de diferentes localidades (Estados de Sergipe e Bahia), coletados em diferentes épocas do ano (inverno e verão) e condições de cultivo (com e sem irrigação) utilizando o perfil cromatográfico obtido por CLAE-DAD combinado com análises quimiométricas.

\section{PARTE EXPERIMENTAL}

\section{Material botânico e reagentes}

Folhas de sete genótipos (106 a 110, 201 e 202) de Lippia gracilis Schauer do Banco Ativo de Germoplasma da Universidade Federal de Sergipe (UFS), localizado na Fazenda Experimental “Campus Rural da UFS", foram colhidas em julho de 2009 (inverno) e janeiro de 2010 (verão com e sem irrigação). Os genótipos com 
código de 106 a 110 são oriundos do estado de Sergipe, enquanto os genótipos com códigos de 201 e 202 são provenientes do estado da Bahia. Exsicatas dos espécimens foram depositadas no Herbário da Universidade Federal de Sergipe, sob os números 14733, 14737, $14734,14735,14732,14736$ e 14731, para os genótipos 106 a 110 , 201 e 202 , respectivamente.

Para compor a fase móvel foram utilizados acetonitrila (Tedia, Fairfield, OH, EUA) e metanol (Tedia) de pureza analítica e grau CLAE, ácido fórmico (88\%) (JT Baker, Philipsburg, PA, EUA) de grau analítico e água purificada em sistema Milli-Q (Millipore, São Paulo, SP, Brasil). Os solventes foram filtrados através de membranas de nylon 0,45 $\mu \mathrm{m}$ (MFS) e desgaseificados utilizando banho ultrassônico da marca Unique (Indaiatuba, SP, Brasil) modelo 1600A.

\section{Preparação das amostras}

O chá de cada um dos sete genótipos (106 a 110, 201 e 202) dos três cultivos [inverno (inv), verão com irrigação (vc) e verão sem irrigação (vs)] de Lippia gracilis foi obtido pelo método de infusão, adicionando-se $2 \mathrm{~g}$ de folhas secas a $200 \mathrm{~mL}$ de água destilada a uma temperatura de $94{ }^{\circ} \mathrm{C}$, aquecida em um forno de micro-ondas da marca Eletrolux (Manaus, AM, Brasil) modelo ME28S, deixado em repouso por $10 \mathrm{~min}$ sem agitação à temperatura ambiente. A solução foi, posteriormente, filtrada, congelada e liofilizada sob as seguintes condições: temperatura $-54{ }^{\circ} \mathrm{C}$ e pressão $79 \mu \mathrm{m} \mathrm{Hg}$ em um liofilizador, marca Liotop (São Carlos, SP, Brasil) modelo L101.

\section{Equipamento e condições cromatográficas}

Foi utilizado um cromatógrafo líquido, marca Shimadzu (Quioto, Japão), modelo Prominence, para eluição gradiente, composto de duas bombas LC-6A, desgaseificador DGU-20A 3 , autoinjetor SIL-10A, detector UV com arranjo de diodos SPD-M20Avp conectado a uma interface CBM 20A. O equipamento foi gerenciado pelo programa LC Solution.

As análises por CLAE foram feitas utilizando solução aquosa ácida ( $0,5 \%$ de ácido fórmico) a $15 \mathrm{mg} \mathrm{mL}^{-1}$ de cada um dos chás liofilizados. Foi utilizada uma coluna com fase estacionária $\mathrm{C}_{18}$ Luna $^{\circledR}(5 \mu \mathrm{m}, 25,0$ $\mathrm{x} 0,46 \mathrm{~cm}$, Phenomenex) acoplada a uma coluna guarda com a mesma fase. A eluição no modo gradiente empregou $\mathrm{MeOH}$ (B) e solução de ácido fórmico $0,5 \%$ (v/v) (A), utilizando as seguintes condições: 5 a $28 \%$ (B) em 14 min, 28 a 35\% (B) em 28 min, 35 a 46\% (B) em 33 min, 46 a $80 \%$ (B) em 20 min, 80 a $100 \%$ (B) em 15 min e $100 \%$ (B) por mais 15 min. Ao término da corrida cromatográfica foi feito um gradiente inverso de 100 a $5 \%$ em 35 min e o tempo de recondicionamento da coluna foi de $75 \mathrm{~min}$. A vazão utilizada foi de $1,0 \mathrm{~mL} \mathrm{~min}^{-1} \mathrm{e}$ em cada análise foram injetados $25 \mu \mathrm{L}$ da solução $\left(15 \mathrm{mg} \mathrm{mL}^{-1}\right)$, sendo cada amostra, 21 no total, analisada em quadruplicata. Os cromatogramas foram registrados em $254 \mathrm{~nm}$, enquanto que os espectros UV foram registrados entre 200 e $400 \mathrm{~nm}$ (Figura 1).

\section{Análise quimiométrica}

Para avaliar as semelhanças e diferenças entre os perfis cromatográficos, foram aplicadas ferramentas quimiométricas exploratórias, através do programa computacional Pirouette (v.4.0, Infometrix, EUA). Antes, porém, foi necessário o pré-tratamento dos dados (alinhamento dos picos) utilizando o algoritmo Correlation Optimised Warping $(\mathrm{COW})^{16}$ e posterior pré-processamento (centrado na média) dos dados originais. O pré-tratamento dos dados foi realizado com o programa computacional Matlab (versão 2009a, The MathWorks, Natick, MA, EUA) e as rotinas para o alinhamento estão disponíveis no site do Prof. Rasmus Bro (http://www.models.kvl.dk/algorithms).
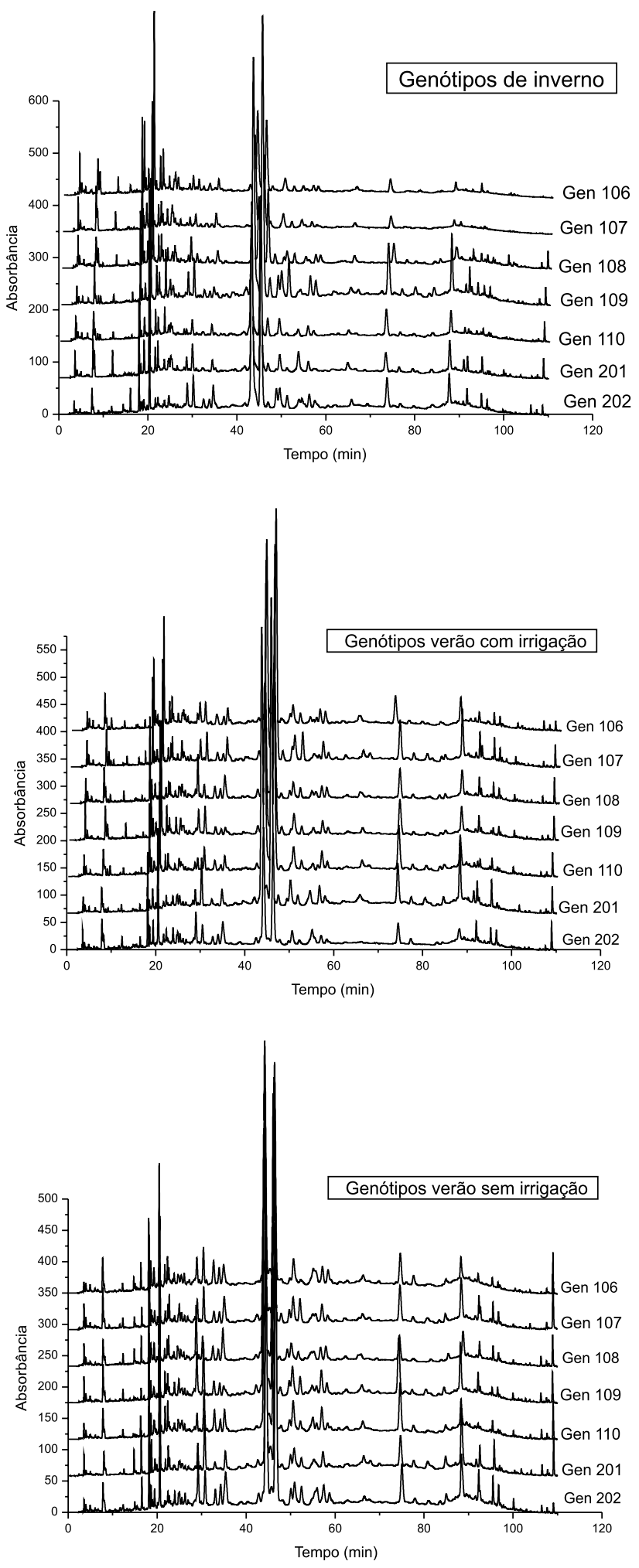

Figura 1. Perfis cromatográficos dos chás dos genótipos de Lippia gracilis, registrados em $254 \mathrm{~nm}$

\section{RESULTADOS E DISCUSSÃO}

\section{Otimizações das condições cromatográficas}

Sabendo que o conteúdo de metabólitos secundários em uma planta pode ser afetado por sua carga genética, ciclo biológico, saúde e sua relação com o meio ambiente (temperatura, disponibilidade hídrica, 
exposição à radiação ultravioleta, altitude, composição atmosférica, solo, entre outras) e que esta variação pode alterar seus efeitos terapêuticos, torna-se viável, para uma caracterização completa da planta estudada, uma análise total dos seus constituintes químicos. ${ }^{12,13,17-22}$

Assim, para obtenção de um perfil cromatográfico representativo, que apresente uma boa separação dos picos, tanto quanto possível, dentro de um tempo de análise razoável, a composição da fase móvel (tipo de solvente e a sua concentração inicial), forma de gradiente de eluição, vazão, fases estacionárias e volume de injeção foram avaliados e otimizados, permitindo alcançar a melhor condição de análise mencionada no item Equipamento e condições cromatográficas. ${ }^{23-25}$ O comprimento de onda selecionado para detecção foi $254 \mathrm{~nm}$, onde se observa o maior número de picos detectáveis.

\section{Análise de componentes principais}

Observando a Figura 1, vê-se significativa semelhança entre os cromatogramas de todas as amostras analisadas. Assim, identificar eventuais diferenças entre elas, apenas com análise visual, torna-se difícil. Neste caso, a aplicação de técnicas quimiométricas fornece a interpretação dos dados com mais qualidade, possibilitando a classificação e a discriminação entre as amostras. ${ }^{26,27}$

Os perfis cromatográficos obtidos foram organizados em uma matriz de dados, contendo 84 linhas (amostras) e 10.314 variáveis (tempos de leitura, 110 min), sendo submetidos à análise de componentes principais (ACP), após pré-tratamento (alinhamento dos picos pela técnica do algoritmo Correlation Optimised Warping -COW) ${ }^{16}$ e pré-processamento (centrados na média) dos dados originais. $\mathrm{O}$ alinhamento é necessário devido a desvios mínimos de tempos de retenção, originados de pequenas oscilações no sistema cromatográfico, que precisam ser removidos para que a análise multivariada se sustente apenas nos tempos de retenção e intensidade relativa das bandas cromatográficas (propriedades inerentes das amostras). A utilização dos dados não alinhados implicaria, por exemplo, na utilização de mais componentes principais do que aquelas realmente necessárias, podendo levar a interpretações equivocadas dos resultados. Outro fator a ser levado em conta é que este desalinhamento não é regular em toda a faixa de tempo analisada e foram observados oscilações de 0,10 a 0,30 min em sinais cromatográficos com uma largura de pico de $2 \mathrm{~min}$.

Analisando o gráfico de escores (Figura 2), observa-se que as componentes principais CP1 e CP2 descrevem 83,6\% da variância total dos dados $(\mathrm{CP} 1=79,3 \%$ e $\mathrm{CP} 2=4,3 \%)$. Convencionando que as amostras projetadas entre $-0,5$ e $+0,5$, tanto em $\mathrm{CP} 1$ quanto em $\mathrm{CP} 2$, foram consideradas próximas de zero, a Figura 2 mostra que em CP1 há agrupamento das amostras 109inv, 202inv, 201vc, 109vs, 110vs, 201vs e 202vs em valores de escores positivos; das amostras 106inv, 107inv, 108inv, 110inv, $108 \mathrm{vc}, 202 \mathrm{vc}$ em valores de escores negativos; das amostras 201inv, 106vc, 107vc, 109vc, 110vc, 106vs, 107vs, 108vs em valores próximos de zero.

Analisando as amostras em CP2, é possível observar a separação das amostras 108inv, 108vc, 201vc, 202vc, 108vs e 202vs em valores de escores positivos; das amostras 106inv, 107inv, 201inv, 109vc, $110 \mathrm{vc}, 109 \mathrm{vs}, 110 \mathrm{vs}$ e $201 \mathrm{vs}$ em valores de escores negativos e, das amostras 109inv, 110inv, 202inv, 106vc, 107vc, 106vs e 107vs em valores próximos de zero. Vale mencionar que as amostras $106 \mathrm{vc}$, $107 \mathrm{vc}, 106 \mathrm{vs}$ e $107 \mathrm{vs}$ apresentaram valores próximos de zero tanto no eixo da CP1 como na CP2.

Através da Figura 2 pode-se observar a separação das amostras e formação de alguns grupos. Um grupo foi formado pelas amostras $108 \mathrm{inv}, 108 \mathrm{vc}$, 108vs e $202 \mathrm{vc}$; outro grupo é formado por $106 \mathrm{vc}$ e $109 \mathrm{vc}$; um terceiro grupo apresenta as amostras 109inv, 202inv e $201 \mathrm{vc}$; um quarto grupo apresenta $107 \mathrm{vc}, 110 \mathrm{vc}, 106 \mathrm{vs}, 107 \mathrm{vs}, 109 \mathrm{vs}$,

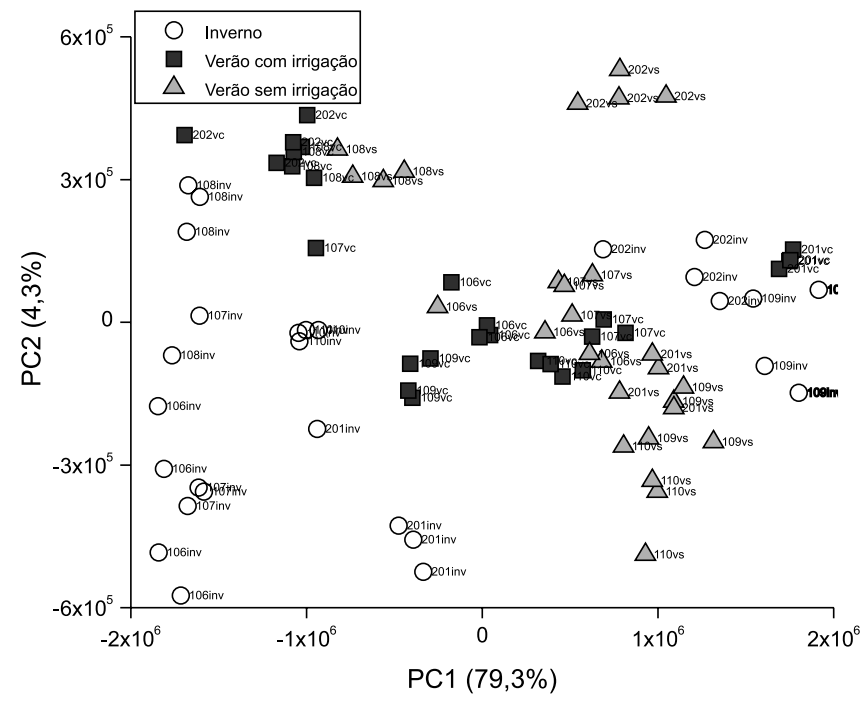

Figura 2. Gráfico de escores (CP1 versus $C P 2$ )

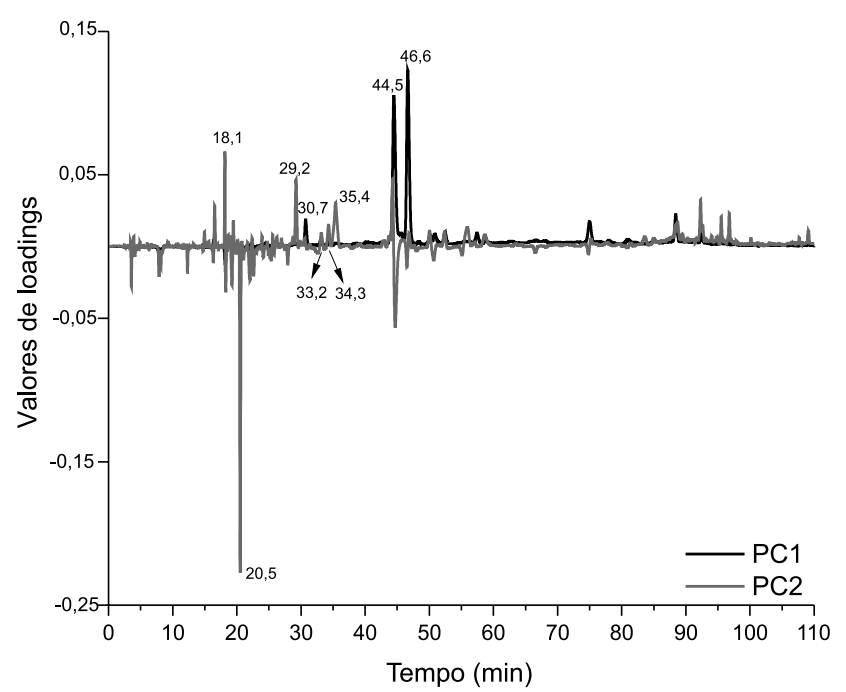

Figura 3. Gráfico de loadings (CP1 versus CP2) das amostras de Lippia gracilis

110vs e 201vs; além de um quinto grupo contendo 106inv e 107inv.

Vale a pena mencionar que os genótipos 108 e 202 coletados no verão e cultivados sem irrigação mostraram ser significativamente diferentes dos demais genótipos da mesma coleta, pois formaram grupos separados (com valores mais positivos em CP2).

Além disso, os genótipos 107, 108 e 110 não se diferenciaram quanto ao tipo de cultivo (verão com e sem irrigação), sendo que o genótipo 108 não foi possível diferenciar pela época da coleta (verão e inverno).

A diferenciação entre as amostras pode ser explicada observando o gráfico de loadings (Figura 3) que demonstra a importância (peso) que cada variável teve na construção das duas componentes principais (CP1 e CP2) e, consequentemente, na projeção das amostras no gráfico de escores.

No gráfico de loadings (Figura 3) é possível perceber que na $\mathrm{CP} 1$ e CP2 algumas bandas cromatográficas se destacam com valores de loadings positivos e outras com valores de loadings negativos. Somente a CP2 apresenta valores de loadings positivos e negativos, enquanto a CP1 só tem valores positivos.

A projeção das amostras 109inv, 202inv, 201vc, 109vs, 110vs, 201vs e 202vs, que apresentaram valores positivos em CP1, está 
relacionada com variáveis que apresentam valores positivos na CP1 no gráfico de loadings (Figura 3), mais especificamente aos picos com tempos de retenção em 44,5 e 46,6 min.

Os loadings da CP2 contêm variáveis com valores positivos e negativos. Sendo assim, para as amostras 106inv, 107inv, 201inv, 109vc, $110 \mathrm{vc}, 109 \mathrm{vs}, 110 \mathrm{vs}$ e $201 \mathrm{vs}$, que apresentaram valores negativos para $\mathrm{CP} 2$, os picos cromatográficos que correspondem às variáveis com valores negativos em CP2 no gráfico de loadings apresentam maior intensidade (Figura 3), sendo representadas, principalmente, pelos picos em 20,5 e 44,5 min. Da mesma forma, a projeção das amostras $108 \mathrm{inv}, 108 \mathrm{vc}, 201 \mathrm{vc}, 202 \mathrm{vc}, 108 \mathrm{vs}$ e $202 \mathrm{vs}$ em valores de escores positivos para $\mathrm{CP} 2$ pode ser relacionada com os tempos de retenção característicos em 18,1; 29,2 e 35,4 min.

No gráfico de escores (Figura 2) percebe-se que, excetuando os genótipos 109 e 202, as demais amostras de inverno apresentam valores em CP1 negativos, tendendo a valores positivos para as amostras coletadas no verão. Estes dados, combinados com aqueles do gráfico de loadings (Figura 3), permite-nos propor que os metabólitos que eluem nos tempos de retenção 44,5 e 46,6 min são mais característicos das amostras coletadas no verão (amostras 106 a 108; 110 e 201). Comparando os respectivos espectros UV destas bandas (Figura 4) com os da literatura ${ }^{28} \mathrm{e}$ o relato da presença de flavonoides em extrato polar de folhas desta espécie, ${ }^{7}$ sugere-se que estes sejam flavonoides glicosilados, o que corrobora a função destes metabólitos como protetores de radiação UV. ${ }^{29}$ Além disso, observou-se também que a irrigação influencia significativamente o perfil cromatográfico
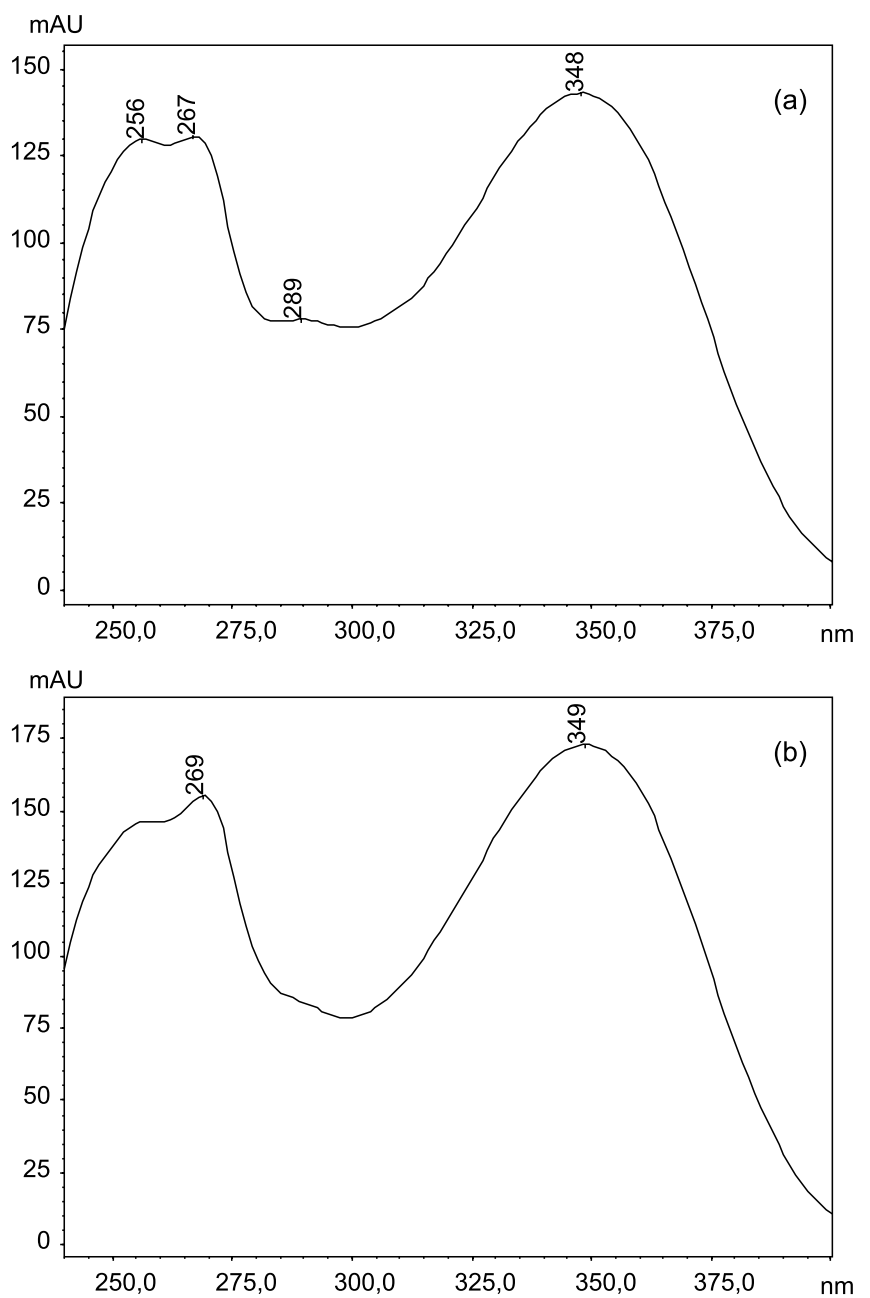

Figura 4. Espectros $U V$ das bandas cromatográficas com tempos de retenção 44,5 $\min ($ a) e 46,6 $\min (b)$ nas amostras 109 e 202, pois reduz as bandas cromatográficas que definem a CP1 (Figura 3).

Sabendo-se do interesse farmacológico nesta planta ${ }^{30}$ e das inúmeras atividades biológicas que os flavonoides apresentam, ${ }^{31}$ a partir de nossos resultados, deve-se considerar as condições de cultivo, época de coleta e seleção do melhor genótipo para sua utilização.

\section{CONCLUSÕES}

Neste trabalho relata-se a obtenção de perfis cromatográficos por CLAE-DAD juntamente com análises quimiométricas, para demonstrar a variação dos componentes químicos entre os chás de folhas de genótipos de L. gracilis oriundos de diferentes localidades (Estados de Sergipe e Bahia) e coletados em diferentes épocas do ano (verão e inverno) e sob diferentes formas de cultivo (verão com e sem irrigação).

A metodologia proposta mostrou ser adequada e eficiente para determinar as diferenças e semelhanças entre as amostras.

Sabendo que esta espécie possui grande resistência à seca e a altas temperaturas, pode-se propor, pelos resultados obtidos, que os genótipos 107, 108 e 110 apresentam maior resistência ao estresse hídrico, pois não se observou diferenciação entre suas amostras coletadas no verão e cultivadas com e sem irrigação. Além disso, o genótipo 108 apresenta pouca diferenciação entre a amostra coletada no inverno e suas correspondentes coletadas no verão, sob as duas formas de cultivo.

Acreditamos que, com este trabalho, se tornou evidente que é preciso considerar as condições de cultivo, época de coleta e seleção do melhor genótipo para utilização de L. gracilis, podendo, portanto, auxiliar na busca racional de metabólitos de interesse para esta espécie.

\section{AGRADECIMENTOS}

Ao CNPq, à CAPES, FAPITEC/SE e RENORBIO pelo apoio financeiro e pelas bolsas concedidas.

\section{REFERÊNCIAS}

1. Melo, J. I. M.; Alves, I. M.; Sousa, R. T. M.; Barbosa, L. M. M. A.; Andrade, W. M.; Revista Caatinga 2010, 23, 41.

2. Pascual, M. E.; Slowing, K.; Carretero, E.; Mata, D. S.; Villar, A.; J. Ethnopharmacol. 2001, 76, 201.

3. Botelho, M. A.; Nogueira, N. A. P.; Bastos, G. M.; Fonseca, S. G. C.; Lemos, T. L. G.; Matos, F. J. A.; Montenegro, D.; Heukelbach, J.; Rao, V. S.; Brito, G. A. C.; Braz. J. Med. Biol. Res. 2007, 40, 349.

4. Lorenzi, H.; Matos, F. J. A.; Plantas Medicinais no Brasil: Nativas e Exóticas, $2^{a}$ ed., Plantarum: Nova Odessa, 2008, p. 527.

5. Albuquerque, U. P.; Medeiros, P. M.; Almeida, A. L. S.; Monteiro, J. M.; Neto, E. M. F. L.; Melo, J. G.; Santos, J. P.; J. Ethnopharmacol. 2007, $114,325$.

6. Gomes, S. V. F.; Santos, A. D. C.; Moraes, V. R. S.; Martins, L. R. R.; Viana, M. D.; Blank, A. F.; Pereira-Filho, E. R.; Cass, Q. B.; Nogueira, P. C. L.; Alves, P. B.; Chromatographia 2010, 72, 275.

7. Guimarães, A. G.; Gomes, S. V. F.; Moraes, V. R. S.; Nogueira, P. C. L.; Ferreira, A. G.; Blank, A. F.; Santos, A. D. C.; Viana, M. D.; Silva, G. H.; Quintans Júnior, L. J.; J. Nat. Med. 2012, 66, 428.

8. Bitu, V.; Botelho, M. A.; da Costa, J. G. M.; Rodrigues, F. F. G.; Veras, H. N. H.; Martins, K. T.; Lyra, A.; Coluchi, G. G.; Ruela, R. S.; Queiroz, D. B.; Siqueira, J. S.; Quintans-Junior, L. J.; Rev. Bras. Farmacogn. 2012, 22, 69; Guilhon, C. C.; Raymundo, L. J. R. P.; Alviano, D. S.; Blank, A. F.; Arrigoni-Blank, M. F.; Matheus, M. E.; Cavalcanti, S. C. H.; Alviano, C. S.; Fernandes, P. D.; J. Ethnopharmacol. 2011, 135, 
406; Cavalcanti, S. C. H.; Niculau, E. S.; Blank, A. F.; Câmara, C. A. G.; Araújo, I. N.; Alves, P. B.; Bioresour. Technol. 2010, 101, 829; Neto, R. M.; Matos, F. J. A.; Andrade, V. S.; Melo, M. C. N.; Carvalho, C. B. M.; Guimarães, S. B.; Pessoa, O. D. L.; Silva, S. L.; Silva, S. F. R.; Vasconcelos, P. R. L.; Rev. Bras. Farmacogn. 2010, 20, 261; Mendes, S. S.; Bomfim, R. R.; Jesus, H. C. R.; Alves, P. B.; Blank, A. F.; Estevam, C. S.; Antoniolli, A. R.; Thomazzi, S. M.; J. Ethnopharmacol. 2010, 129, 391; Teles, T. V.; Bonfim, R. R.; Alves, P. B.; Blank, A. F.; Jesus, H. C. R.; Quintans-Jr, L. J.; Serafini, M. R.; Bonjardim, L. R.; Araujo, A. A. S.; Afr. J. Biotecnol. 2010, 9, 8800; Neves, I. A.; Oliveira, J. C. S.; Camara, C. A. G.; Schwartz, M. O. E.; J. Essent. Oil Res. 2008, 20, 157; Lima, M. C. L.; Lemos, T. L. G.; Pessoa, O. D. L.; Santiago, G. M. P.; Matos, F. J. A.; Arriaga, A. M. C.; de Oliveira, J. P. P.; Sant'ana, A. E. G.; Chem. Nat. Comp. 2008, 44, 254; de Albuquerque, C. C.; Camara, T. R.; Mariano, R. L. R.; Willadino, L.; Marcelino Júnior, C.; Ulisses, C.; Braz. Arch. Biol. Technol. 2006, 49, 527; Santiago, G. M. P.; Lemos, T. L. G.; Pessoa, O. D. L.; Arriaga, A. M. C.; Matos, F. J. A.; Lima, M. A. S.; Santos, H. C.; Lima, M. C. L.; Barbosa, F. G.; Luciano, J. H. S.; Silveira, E. R.; de Menezes, G. H. A.; Nat. Prod. Commun. 2006, 1, 573; Pessoa, O. D. L.; de Carvalho, C. B. M.; Silvestre, J. O. V. I.; Lima, M. C. L.; Neto, R. M.; Matos, F. J. A.; Lemos, T. L. G.; Fitoterapia 2005, 76,712 .

9. Gomes, S. V. F.; Nogueira, P. C. L. N.; Moraes, V. R. S.; Ecl. Quim. 2011, 36, 64.

10. Martins, L. R. R.; Pereira, E. R. F.; Cass, Q. B.; Anal. Bioanal. Chem. 2011, 400, 469 .

11. Jiang, Y.; David, B.; Tu, P.; Barbin, Y.; Anal. Chim. Acta 2010, 657, 9.

12. Wang, Y.; Han, T.; Zhang, X.-G.; Zheng, C.-J.; Rahman, K.; Qin, L. P.; Chromatographia 2009, 70, 143.

13. Chen, J.; Lu, Y.-H.; Wei, D.-Z.; Zhou, X.-L.; Chromatographia 2009, $70,981$.

14. Zhao, C.; Chan, H.-Y.; Yuan, D.; Liang, Y.; Lau, T.-Y.; Chau, F.-T.; Phytochem. Anal. 2011, 22, 503.

15. Morgano, M. A.; Queiroz, S. C. N.; Ferreira, M. M. C.; Braz. J. Food Technol. 1999, 2, 73.
16. Skov, T.; Berg, F. V. D.; Tomasi, G.; Bro, R.; J. Chemom. 2006, 20, 484.

17. Zhou, X.; Zhang, Y.-S.; Zhao, Y.; Gong, X.-J.; Zhao, C.; Chen, H.-G.; Chromatographia 2009, 69, 1282.

18. Yang, J.; Chen, L.-H.; Zhang, Q.; Lai, M.-X.; Wang, Q.; J. Sep. Sci. 2007, 30, 1276.

19. Gobbo-Neto, L.; Lopes, N. P.; Quim. Nova 2007, 30, 274.

20. Garza-Juárez, A.; Salazar-Cavazos, M. L.; Salazar-Aranda, R.; PérezMeseguer, J.; Torres, N. W.; Planta Med. 2011, 77, 958.

21. Han, C.; Chen, J.; Chen, B.; Lee, F. S.-C.; Wang, X.; J. Sep. Sci. 2006, 29, 2197.

22. Liu, X.-J.; Hu, J.; Li, Z.-Y.; Qin, X.-M.; Zhang, L.-Z.; Guo, X.-Q.; Arch. Pharm. Res. 2011, 34, 961.

23. Ji, Y.-B.; Xu, Q.-S.; Hu, Y.-Z.; Heyden, Y. V.; J. Chromatogr., A 2005 , 97, 1066.

24. Xie, P.; Chen, S.; Liang, Y.-Z.; Wang, X.; Tian, R.; Upton, R.; J. Chromatogr, A 2006, 171, 1112.

25. Snyder, L. R.; Dolan, J. W.; J. Chromatogr., A 1996, 721, 3.

26. Mok, D. K. W.; Chau, F.-T.; Chemom. Intell. Lab. Syst. 2006, 82, 210.

27. Li, B.-Y.; Hu, Y.; Liang, Y.-Z.; Xie, P.-S.; Du, Y.-P.; Anal. Chim. Acta 2004, 514, 69.

28. Ducrey, B.; Wolfender, J. L.; Marston, A.; Hostettmann, K.; Phytochemistry 1995, 38, 129.

29. Sisa, M.; Bonnet, S. L.; Ferreira, D.; van der Westhuizen, J. H.; Molecules 2010, 15, 5196.

30. http://bvsms.saude.gov.br/bvs/publicacoes/fitoterapia_no_sus.pdf, acessada em Agosto 2012.

31. Keller, R. B., ed.; Flavonoids: Biosynthesis, Biological Effects and Dietary Sources, Nova Science Publ.: New York, 2009; Atmani, D.; Chaher, N.; Berboucha, M.; Debbache, N.; Boudoud, H.; Curr. Nutr. Food Sci. 2009, 5, 225; Yao, L. H.; Jiang, J.-S.; Tomás-Barberán, F. A.; Datta, N.; Singanusong, R.; Chen, S. S.; Plant Foods Hum. Nutr. 2004, 59, 113. 